PAWEL ŁAPIŃSKI

ORCID: 0000-0003-2220-3485

Université de Gdańsk

pawel.lapinski@ug.edu.pl

\title{
PEUT-ON VENDRE LA PÉRIPHÉRICITÉ ? OBSERVATIONS SUR LES PÉRITEXTES ÉDITORIAUX DES ROMANS POLONAIS TRADUITS EN FRANÇAIS
}

\section{INTRODUCTION}

Les chercheurs, tels Johann Heilbron, Gisèle Sapiro ou Pascale Casanova, décrivent la circulation globale des biens littéraires comme un système strictement hiérarchisé ${ }^{1}$. Bien qu'ils utilisent pour ce faire des termes différents, leurs visions ne laissent aucun doute quant au déséquilibre des échanges littéraires entre le centre et les périphéries. Ainsi, les langues centrales, ou dominantes ${ }^{2}$, peu nombreuses (l'anglais, le français et l'allemand), sont de grandes exportatrices, tandis que les langues semi-périphériques et périphériques, dominées, importent surtout les littératures du centre et peinent à exporter les leurs. Ceci est parfaitement illustré par la position de la littérature polonaise en France. Bien que le nombre de traductions ait récemment connu une augmentation assez importante, la part de marché occupée par la littérature polonaise reste marginale : elle était, en 2018 , de $0,7 \%$ contre,

${ }^{1}$ Cf. P. Casanova, «Consécration et accumulation de capital littéraire », Actes de la Recherche en Sciences Sociales 144, septembre 2002, pp. 7-20 ; J. Heilbron, « Book Translation as a Cultural World-System », European Journal of Social Theory 2(4), 1999, pp. 429-444 ; G. Sapiro, « Translation as a Weapon in the Struggle Against Cultural Hegemony in the Era of Globalization », Bibliodiversity 3, 2014 (<https://www.alliance-editeurs.org/IMG/pdf/bibliodiversity_3_sapiro-2.pdf> [consulté le 30.04.2020]).

2 Pour les définitions des langues centrales et périphériques, voir J. Heilbron, op. cit., et pour les définitions des langues dominantes et dominées, P. Casanova, op. cit. 
par exemple, $1,9 \%$ pour la littérature traduite du russe ou $4,5 \%$ pour la littérature traduite de l'italien ${ }^{3}$.

Ainsi, on peut constater que les auteurs polonais et leurs œuvres restent plutôt mal connus du lectorat français. Cette méconnaissance peut motiver une stratégie d'exotisation ${ }^{4}$, si l'éditeur décide de souligner les origines de l'auteur ou de l'œuvre en accentuant le fait qu'ils représentent une littérature peu présente sur le marché français et donc périphérique selon la classification proposée par Heilbron. Or, l'exotisation, en tant que procédé éditorial, peut jouer un rôle dans la création d'une marque littéraire, aujourd'hui l'un des éléments essentiels du marketing littéraire ${ }^{5}$.

L'objectif du présent article sera de vérifier si les éditeurs français de littérature polonaise adoptent la stratégie d'exotisation pour vendre leurs livres. Pour répondre à cette question, nous analyserons le cas de quatre maisons d'édition dont les titres polonais ont gagné une certaine reconnaissance, attestée notamment par l'attribution de prix littéraires. Nous vérifierons si les paratextes qu'elles présentent aux lecteurs se réfèrent explicitement ou implicitement à l'origine périphérique des auteurs ou des œuvres. De telles pratiques ont été observées dans le cas d'autres relations de type centre-périphérie, notamment dans le domaine post-colonial ${ }^{6}$.

Notre corpus de recherche est composé de livres publiés entre 2008 et 2018 par deux maisons d'éditions actives depuis longtemps et bien implantées sur le marché, à savoir Noir sur Blanc et Actes Sud, et deux autres fondées relativement récemment, Mirobole et Agullo. Les maisons d'éditions choisies se trouvent parmi les promoteurs les plus actifs de la littérature polonaise dans l'Hexagone dans la période susmentionnée ${ }^{7}$, et dès lors le corpus peut être considéré comme représentatif.

${ }^{3}$ Observatoire de l'économie du livre du Service du livre et de la lecture de la Direction générale des médias et des industries culturelles (DGMIC), « Chiffres-clés du secteur du livre 2017-2018» $(<$ https://www.culture.gouv.fr/Sites-thematiques/Livre-et-Lecture/Actualites/Chiffres-cles-dusecteur-du-livre-2017-20182> [consulté le 30.04.2020]).

${ }^{4}$ Il s'agirait ici de l'exotisme conçu comme une manière de représenter ou voir l'Autre qui reste asymétrique puisqu'elle présuppose «l'étrangeté d'un objet aux yeux du sujet par défaut "normal", "habituel" » (cf. M. Maiatsky, « Comme dans le ventre de sa marâtre », Études de lettres 2-3, 2009 (<http://journals.openedition.org/edl/445> [consulté le 30.10.2020]).

5 Cf. G. Huggan, The Postcolonial Exotic. Marketing the margins, Routledge, London-New York 2001 ; S. Debenedetti, «Le marketing des industries culturelles : films, livres et musique enregistrée », [dans :] D. Bourgeon-Renault (dir.), Marketing de l'Art et de la Culture : spectacles vivants, patrimoine et industries culturelles, Dunod 2014, pp. 241-281.

${ }^{6}$ Cf. K. Batchelor, Translation and Paratexts, Routledge, London 2018 ; U. Kluwick, «Postcolonial Literatures on a Global Market. Packaging the "Mysterious East" for Western Consumption », [dans :] P. Rüdiger, K. Gross (dir.), Translation of Cultures, Brill, Amsterdam-New York pp. 75-92; D. Dumontet, « Possibilités et limites des transferts culturels : le cas des romans La Reine Soleil levée de Gérard Étienne et Texaco de Patrick Chamoiseau », TTR : Traduction, Terminologie, Rédaction 13(2), 2000, pp. 149-178.

7 Selon le catalogue de la Bibliothèque nationale de France $(<$ https://catalogue.bnf.fr/index. do $>$ ), dans la période couverte par l'article, environ 320 œuvres littéraires polonaises ont été publiées en France ; 42 titres ont été publiés par Noir sur Blanc, dont la domination parmi les éditeurs 
Nous nous pencherons d'abord sur l'épitexte éditorial qui apporte des informations générales sur la politique déclarée des quatre maisons d'édition en question. Ensuite, nous nous intéresserons à trois éléments du péritexte éditorial, à savoir la collection dont le texte traduit fait partie, la quatrième et la première de couverture. Ces informations nous permettront de reconstruire les stratégies éditoriales. La division de l'offre en collections est destinée à « indiquer immédiatement au lecteur potentiel à quel type, sinon à quel genre d'ouvrage il a affaire $»^{8}$. La quatrième de couverture, «zone du péritexte qui se trouve sous la responsabilité directe et principale (mais non exclusive) de l'éditeur » ${ }^{9}$, transmet, par définition, la vision de ce dernier sur l'œuvre et son auteur ${ }^{10}$. En outre, dans le cas d'une collection, le contenu de plusieurs quatrièmes de couverture lues ensemble peut former une sorte de narration en dialogue avec les principes ou idées directrices sur lesquels celle-ci se fonde ${ }^{11}$. Enfin, la quatrième et la première de couverture jouent ensemble un rôle de marketing essentiel : la première est « l'ambassadrice du texte », qui forge la première impression et encourage à retourner le livre afin de consulter la quatrième ${ }^{12}$, laquelle, à son tour, a pour but d'inciter à l'achat ${ }^{13}$. L'illustration de couverture, en tant que support iconographique, peut être qualifiée de paratexte non-verbal, car elle entre avec le titre dans une relation iconotextuelle caractérisée par une « interdépendance pourvoyeuse de sens » ${ }^{14}$ qui apporte généralement un certain commentaire sur le contenu de l'œuvre.

est indiscutable, et 11 par Actes Sud qui se place parmi les cinq premiers. Quant à Mirobole (fondée en 2011) et Agullo (fondée en 2015), elles opèrent sur le marché depuis moins longtemps et sont plus petites, mais dès le début, elles ont manifesté un grand intérêt pour la littérature polonaise et méritent donc d'être incluses dans l'analyse.

${ }^{8}$ G. Genette, Seuils, Seuil, Paris 2002, p. 25.

9 Ibidem, p. 20.

10 Étant donné qu'une part de plus en plus considérable des achats de livres se fait sur Internet, nous prendrons aussi en considération les notes et notices biographiques publiées sur les sites web des éditeurs.

11 Cf. E. Skibińska, «Traduire par temps de ruptures, ou comment la collection Pavillons/ Domaine de l'Est (Robert Laffont, 1980-2003) a orienté l'image de la littérature est-européenne en France », [dans :] M. Laurent (dir.), Traduction et Rupture. La traduction comme moyen de communication interculturelle, Éditions Numilog, Paris 2014, pp. 259-275.

12 A. Grenon, «La littérature young adult en France : quand le marketing s'empare du processus éditorial. Comment les diverses maisons d'édition concernées s'approprient-elles ce phénomène? », Sciences de l'information et de la communication, Département Documentation, Archives, Médiathèque et Édition, Université Toulouse Jean Jaurès, 2017, p. $25(<\mathrm{https}$ ://dumas.ccsd.cnrs.fr/ dumas-01858699/document> [consulté le 30.04.2020]).

13 Cf. A. Haslam, Book Design, Laurence King Publishing, London 2006, p. 161.

${ }^{14}$ C. Cua, Représenter et traduire la culture à travers l'image : la Chine et le Japon dans l'album illustré jeunesse en France et au Québec (2000-2015), Université York, Toronto, Ontario 2019, p. 15 (<https://yorkspace.library.yorku.ca/xmlui/bitstream/handle/10315/36322/Cua_Catherine_2019_ PHD.pdf? sequence=2\&isAllowed=y $>$ [consulté le 30.04.2020]). 


\section{NOIR SUR BLANC}

Nous nous pencherons tout d'abord sur la maison d'édition Noir sur Blanc (NsB), probablement la plus renommée en matière de littérature polonaise, qui domine largement les autres entités de ce cercle restreint par le nombre de titres publiés. Sa politique éditoriale consiste à « créer des passerelles entre les cultures, [...] donner une image toujours plus fidèle du bouillonnement culturel observé à l'Est de l'Europe $»^{15}$. Les éditeurs se voient comme "passeurs de textes entre le monde francophone et la Pologne, et au-delà, les pays de l'Est européen $»^{16}$. Il apparait donc clairement que toute son activité est fondée sur l'idée d'offrir aux lecteurs du centre des œuvres représentatives des périphéries. En effet, NsB publie les auteurs polonais les plus célèbres à l'heure actuelle, lauréats de prix majeurs du champ littéraire polonais : Dorota Masłowska, Eustachy Rylski, Andrzej Bart, Olga Tokarczuk, Jacek Dehnel, Joanna Bator, Szczepan Twardoch et bien d'autres. Néanmoins, bien que la littérature polonaise occupe une place prépondérante dans l'offre de l'éditeur et malgré l'intention déclarée de « créer des passerelles » entre l'Est et la France, la périphéricité des auteurs est rarement utilisée en tant que telle pour attirer l'attention du lecteur. La division du catalogue en séries est relativement simple et basée sur les genres : Littérature (divisée entre Littérature française et Littérature étrangère), Essais-Documents, Poésie et Beaux Livres.

Les références à l'origine des auteurs sont également rares sur la quatrième de couverture. L'exotisme de la périphérie y apparaît parfois, mais plutôt en tant qu'élément du résumé que comme outil de marketing. C'est notamment le cas pour les œuvres du reporter Mariusz Wilk, qui «s'aventure dans les antichambres de l'autre monde $»^{17}$, insuffle aux lecteurs « la passion des grands espaces $»^{18}$ ou encore « interpelle l'homme occidental sur sa manière de vivre $»^{19}$. La seule note contenant des notions explicitement exotisantes est celle de Drach de Szczepan Twardoch, où la Silésie, région qui sert de toile de fond au récit, est décrite comme une « terre méconnue, mystérieuse, âpre » qui se révèle « un écheveau d'histoires, de peuples et de langues $»^{20}$. À l'inverse, le critère selon lequel l'éditeur a composé son offre est bien visible dans les notices biographiques, qui décrivent le plus souvent les auteurs d'après leur position dans le champ littéraire polonais.

$15<\mathrm{http}: / /$ www.leseditionsnoirsurblanc.fr/historique> [consulté le 30.04.2020].

16 Ibidem.

$17<\mathrm{http} / /$ www.leseditionsnoirsurblanc.fr/dans-les-pas-du-renne-mariusz-wilk-978288 2502209> [consulté le 30.04.2020].

$18<$ http://www.leseditionsnoirsurblanc.fr/portage-mariusz-wilk-9782882502353> [consulté le 30.04.2020].

$19<\mathrm{http}$ //www.leseditionsnoirsurblanc.fr/la-maison-du-vagabond-mariusz-wilk-978288250 4081> [consulté le 30.04.2020].

$20<$ http://www.leseditionsnoirsurblanc.fr/drach-szczepan-twardoch-9782882505132> [consulté le 30.04.2020]. 
Wojciech Tochman est «l'un des chefs de file de l'école polonaise du reportage littéraire », Jacek Dehnel « considéré en Pologne comme l'un des écrivains les plus talentueux de la jeune génération », ou Dorota Masłowska, « l'une des voix les plus percutantes de la littérature polonaise contemporaine ». Olga Tokarczuk est la « romancière polonaise la plus traduite à travers le monde », Szczepan Twardoch "poursuit en Pologne une œuvre singulière ». Ainsi, NsB se présente en éditeur des meilleurs représentants de la littérature polonaise du moment, ce qui s'inscrit dans ses déclarations à propos de sa ligne éditoriale.

Les couvertures de NsB révèlent une tendance à utiliser des reproductions de peintures et photographies la plupart du temps conformes à l'intrigue. Elles remplissent ainsi un rôle situé entre l'illustration et le résumé. Tel est le cas, par exemple, des couvertures de romans historiques dont l'action se déroule à l'époque napoléonienne (tableau représentant des soldats pour La Condition d'Eustachy Rylski) ou en Espagne au début du XIX ${ }^{\mathrm{e}}$ siècle (tableau de Goya pour Saturne de Jacek Dehnel, qui est consacré à la famille de l'artiste espagnol). Un modèle similaire apparaît sur les couvertures d'autres œuvres dont l'intrigue se situe dans des temps révolus, comme Sonia d'Ignacy Karpowicz, dont l'histoire se passe principalement dans l'entre-deux-guerres et pendant la Seconde Guerre mondiale (photographie en noir et blanc représentant une femme en tenue d'époque) ou La Belle Jeunesse de Marek Hłasko (photographie de jeunes gens sur un manège, qui laisse deviner, par leurs vetements, qu'il s'agit des années 1950, époque dépeinte dans l'œuvre). Cette règle s'applique enfin aussi aux œuvres de Mariusz Wilk, dont les couvertures sont ornées de différentes photographies représentant des paysages et animaux de la Russie rurale décrite par l'auteur. Pour un autre groupe de livres, un peu moins nombreux, l'éditeur a également recours à des peintures et photographies qui s'inscrivent dans la diégèse de l'œuvre, mais de manière moins illustrative et plus symbolique ; elles ne permettent pas au lecteur d'identifier immédiatement le lien entre la couverture et le contenu. Tel est le cas, par exemple, de Drach de Szczepan Twardoch (photographie en noir et blanc d'un chasseur dont la tenue suggère l'époque de la Grande Guerre, ce qui correspond à certaines trames de l'intrigue), ou L'Estivant de Kazimierz Orłoś (peinture coloriste de Józef Czapski représentant deux femmes dans la mer, référence à un moment essentiel du livre, et correspondant en même temps au titre). À ceci s'ajoutent les couvertures métaphoriques, qui intriguent le lecteur sans laisser réellement deviner leur relation avec le contenu, comme celle des Pérégrins d'Olga Tokarczuk, qui figure les cours des plus grands fleuves du monde. Dans quelques cas enfin, nous pourrions être portés à parler d'une forme de traduction intersémiotique, où ce n'est pas la diégèse qui est représentée, mais le style de l'œuvre. C'est le cas de la couverture de Morphine de Twardoch, dont la couverture blanche donne à voir un dessin expressif, en noir, qui véhicule l'ambiance angoissante et la narration schizophrénique du roman, ou de celle de Jul de Paweł Goźliński, qui représente un crâne de facture étrange, avec des tentacules en lieu et place de la mâchoire 
— sans doute une référence à l'atmosphère gothique qui règne dans ce roman policier historique.

En conclusion, nous pouvons constater que, malgré la ligne éditoriale de $\mathrm{NsB}$, qui repose sur l'idée d'une communication littéraire entre la France et l'Europe centrale, voire orientale, l'éditeur évite généralement dans son paratexte d'attribuer à ses auteurs ou à leurs œuvres des caractéristiques exotisantes qui se référeraient à la périphéricité. Étant donné que la maison a été fondée par un « couple aux origines suisses, polonaises, russes et autrichiennes $»^{21}$, Vera et Jan Michalski, cet évitement de la référence à la périphéricité — qui risque toujours d'être quelque peu réductrice - pourrait être interprété comme un signe de respect pour la culture de ces régions de l'Europe.

\section{ACTES SUD}

Parmi tous les éditeurs qui publient régulièrement des traductions du polonais, Actes Sud est, pour le moment, le seul avec Gallimard à être classé parmi les plus grands acteurs du marché ${ }^{22}$. Cette maison développe « une politique éditoriale généraliste » qui se caractérise dès l'origine par « une large ouverture aux littératures étrangères $»^{23}$. Elle compte même dans son catalogue plusieurs séries régionales, dont certaines dédiées à la littérature de l'Europe centrale et orientale (Lettres balkaniques, Lettres hongroises et Lettres russes). Actes Sud a publié peu de littérature polonaise et semble suivre peu ou prou la même règle que Noir sur Blanc, qui consiste à sélectionner avant tout les œuvres et auteurs récompensés par des prix littéraires en Pologne. La liste des traductions du polonais comprend des œuvres de Mariusz Szczygieł (Gottland, Chacun son paradis), Wiesław Myśliwski (L'Art d'écosser les haricots, La dernière partie), Andrzej Stasiuk (L'Est, Pourquoi je suis devenu écrivain, Taksim, Un vague sentiment de perte) et Lidia Amejko (La Vie des saints de la Cité). Malgré leurs genres différents, ces titres sont tous classés comme «Littérature / Hors collection ».

La quatrième de couverture présente peu de références à la périphéricité. Les périphéries ne sont mentionnées que dans le cas des reportages de Stasiuk et Szczygieł, ce qui est principalement imputable à la thématique de leurs œuvres (l'ancienne Tchécoslovaquie et l'actuelle République tchèque chez Szczygieł, l'Est de l'Europe et l'Asie chez Stasiuk). Il faut néanmoins noter que l'éditeur ne se contente pas de les évoquer comme une part indispensable du résumé, mais tente

$21<\mathrm{http}: / /$ www.leseditionsnoirsurblanc.fr/historique $>$ [consulté le 30.04.2020].

22 Cf. F. Piault, «Classement 2018. Les 200 premiers éditeurs français », Livres Hebdo 1179, $2018(<\mathrm{https}: / /$ www.csp.fr/sites/default/files/content/press-article/file/1806/livre_hebdo_classement_ juin_2018.pdf> [consulté le 30.04.2020]).

$23<$ https://www.actes-sud.fr/node/63312> [consulté le 30.04.2020]. 
aussi parfois de les mettre en avant comme un élément de l'attractivité du livre en tant qu'objet de lecture. Ainsi, dans la note de Chacun son paradis de Szczygieł, l'éditeur assure que Szczygieł « en foulant les pavés de Prague et des petites villes tchèques $[. .$.$] rend si bien hommage à la laïcité sans faille, à la singularité$ culturelle et à la liberté d'esprit de son pays de prédilection qu'il suscite chez son lecteur l'irrépressible désir de se lancer sur ses traces ». La note de L'Est de Stasiuk s'ouvre sur une constatation arbitraire (« Les pays de 1'Est de l'Europe fascinent et intriguent ») pour ensuite dépeindre l'auteur comme quelqu'un qui « immerge et fait partager » au lecteur « sa vision de l'Est », cette vaste région étant résumée à des « espaces immenses, terres arides, paysages inchangés depuis des siècles ». Les notices biographiques suivent à peu près le même schéma que chez NsB : les auteurs sont présentés à l'aune du champ littéraire national. Myśliwski est ainsi « l'un des écrivains polonais contemporains les plus importants [...], récompensé deux fois par le prix Nike, le plus grand prix littéraire polonais », et Stasiuk, le « chef de file de la nouvelle littérature polonaise ». Néanmoins, quelques notions régionales (transnationales) sont perceptibles lorsque Szczygieł est décrit comme journaliste et rédacteur au « plus grand quotidien indépendant en Europe centrale » (Gazeta Wyborcza) et Stasiuk, comme écrivain caractérisé par un « amour profond pour l'arrière-cour de l'Europe ».

Il serait difficile, concernant les premières de couverture, de parler d'une quelconque cohérence ou, a minima, de conceptions répétitives. Les illustrations varient aussi bien dans leur technique que dans leur relation avec le contenu. Elles incluent des photographies (par exemple, pour L'Est, Gottland et Chacun son paradis), des photographies modifiées (La dernière partie et Taksim), ainsi que des œuvres picturales ou collages (L'Art d'écosser les haricots, Pourquoi je suis devenu écrivain et La Vie des saints de la Cité). Pour certains livres, l'illustration peut facilement être associée au contenu — particulièrement dans les cas de L'Est (photographie de collines herbeuses), Gottland (photographie du pont Charles de Prague) ou La dernière partie (photographie d'une femme en jupe rose « rétro », qui peut figurer une annonce de la rétrospection du narrateur au temps de sa jeunesse). Les autres illustrations reposent soit sur une impression inspirée par la diégèse (collage avec une façade de bâtiment pour La Vie des saints de la Cité, qui se réfère justement à la cité du titre), soit sur une métaphore plus ou moins ambiguë dont la relation avec l'intrigue est quelque peu arbitraire (par exemple, l'image d'un homme tenant un bouquet de ballons qui l'emportent dans le ciel, dans le cas de Pourquoi je suis devenu écrivain, une autobiographie de Stasiuk). La seule couverture à jouer visiblement sur l'exotisme de la périphérie est celle de Chacun son paradis, un recueil de reportages sur la Tchéquie actuelle. Elle consiste en une photo monochrome avec des éléments colorés montrant des personnes en maillot de bain faisant la queue devant le guichet d'un snack-bar, avec, au premier plan, 
une femme corpulente en maillot deux-pièces qui sourit au photographe, tenant fièrement huit grosses chopes de bière.

Pour conclure on peut reconnaître qu'Actes Sud publie de la littérature polonaise assez régulièrement et avec un certain succès - comme le prouvent le Prix du livre européen attribué en 2009 à Szczygieł et le Prix Nicolas Bouvier décerné en 2018 à Stasiuk — mais cela n'est lié à aucun projet éditorial plus vaste. Les auteurs polonais sont publiés hors collections et font partie du catalogue riche et varié de littérature mondiale qui fait la fierté de cette maison d'édition ${ }^{24}$. Le paratexte qui accompagne les œuvres polonaises ne manifeste pas d'intérêt particulier pour leur origine périphérique.

\section{DE NOUVEAUX JOUEURS}

Pour finir, nous nous intéresserons à deux maisons d'édition qui, bien qu'opérant sur le marché depuis moins longtemps que Noir sur Blanc et Actes Sud, ont déjà montré un certain intérêt pour la littérature polonaise. Le choix de les examiner ensemble n'est pas sans fondement, puisque leurs histoires sont liées : Mirobole a été fondée en 2011 par Sophie de Lamarlière et Nadège Agullo, puis cette dernière l'a quittée pour fonder en 2015 sa propre maison d'édition, Agullo. Les deux éditeurs se distinguent de leurs concurrents par un concept graphique très caractéristique et uniforme pour leurs couvertures, qui est l'œuvre du même artiste, Sean Habig. Enfin, leur ligne éditoriale les rattache également.

\section{MIROBOLE}

Mirobole précise d'entrée de jeu qu'elle ne publie que des livres étrangers, ce qui est assez original en France, où la part des traductions dans la production commercialisée de livres imprimés ne dépasse pas $20 \%{ }^{25}$. De plus, la maison invite ses lecteurs à «pénétrer dans des mondes inconnus » et déclare affectionner « les migrants littéraires » (sans préciser ce terme) et aimer « créer des ponts ». Dans le même temps, afin de ne pas laisser le lecteur potentiel sans repères en terre étrangère, elle associe un certain exotisme aux points de référence bien connus que sont les genres, décrivant son offre à l'aide d'étiquettes telles que « la litté-

24 Pour nuancer un peu cette image, il vaut la peine de noter qu'en janvier 2020, Actes Sud a publié Au nom de l'enquête de Marcin Wroński dans sa collection "Actes noirs », ce qui prouve encore une fois que le polar joue aujourd'hui un rôle majeur dans l'échange de biens littéraires à l'échelle mondiale.

25 Observatoire de l'économie du livre du Service du livre et de la lecture de la Direction générale des médias et des industries culturelles (DGMIC), « Chiffres-clés du secteur du livre 2017-2018 ». 
rature d'anticipation russe », « le polar polonais », «l'absurde sauce moldave », «le conte d'épouvante suédois » ou encore «le whodunit version turque $»^{26}$.

Cette idée exploratrice est bien visible dans les noms de trois collections qui déclinent le concept de «l'horizon » : «Horizons noirs » pour la littérature policière, « Horizons pourpres » pour la littérature de l'étrange et « Horizons blancs » pour la fiction sans distinction de genre. Il n'est pas surprenant de constater que les «mondes inconnus » littéraires se situent plus facilement dans les périphéries ; ainsi l'offre de la maison comprend des titres traduits du polonais, du hongrois, du tchèque, du roumain et du russe, mais Mirobole ne se limite pas à un secteur géographique et publie aussi des auteurs suédois, danois, turcs, grecs, espagnols, portugais, néerlandais, chinois et même quelques traductions de l'allemand et de l'anglais. En ce qui concerne les auteurs polonais, nous y trouvons deux livres de Zygmunt Miłoszewski (Les impliqués, Un fond de vérité) inclus dans la collection « Horizon noirs », et deux livres de Marek Hłasko (Converti à Jaffa et $L a$ mort du deuxième chien) intégrés à la collection « Horizons blancs ».

La quatrième de couverture est presque entièrement dominée par les résumés, dont le style est plutôt neutre, bien que dans deux cas l'éditeur tente de présenter les auteurs comme des guides accompagnant le lecteur en «terre inconnue », qu'il s'agisse de la Pologne d'aujourd'hui ou de la république populaire de Pologne d'hier. Ainsi, Un fond de vérité de Miłoszewski est décrit comme « un polar sans concession, qui plonge au cœur de la société et l'histoire polonaise », tandis que Hłasko est montré comme un écrivain qui s'efforce notamment de " décrire au monde de l'Ouest la vie sous le communisme » — affirmation qui semble d'ailleurs peu pertinente étant donné que l'action des deux titres publiés se déroule en Israël dans les années 1960. Nous retrouvons, dans les notices biographiques, le même procédé que chez Noir sur Blanc ou Actes Sud, qui consiste à replacer l'auteur dans le contexte du champ littéraire polonais : Miłoszewski est ainsi « un auteur phare de la jeune génération polonaise ». En revanche, la notice de Hłasko annonce que celui-ci est « comparé à Jack Kerouac pour son approche de la littérature ", ce qui s'inscrit dans la stratégie de l'éditeur — déjà bien visible dans sa déclaration concernant sa ligne éditoriale — d'offrir au lecteur confronté à un écrivain peu connu un point de référence bien ancré dans le paysage littéraire.

Les couvertures constituent l'un des traits caractéristiques de l'offre de Mirobole ; celles-ci sont toutes maintenues dans un même style, non seulement pour la mise en page et la typographie, mais aussi et surtout, pour l'illustration, qui est toujours réduite à un seul objet (couvert par le nom de l'auteur et le titre) dont la relation avec le contenu est plutôt symbolique. Dans le cas de Miłoszewski, il s'agit d'un fauteuil de bureau abîmé de style rétro pour Les impliqués (un clin d'œil probable au fait que l'intrigue opère une rétrospection à l'époque communiste) et une étrange chaise en bois pour Un fond de vérité. Cette dernière est

$26<$ http://mirobole-editions.com/la-maison/> [consulté le 30.04.2020]. 
probablement une référence aux sculptures de chaises vides de la place des Héros du ghetto de Cracovie, symbole des résidents juifs qui ont laissé tous leur biens derrière eux lors de leur déportation, l'antisémitisme étant un leitmotiv du roman. Pour les deux livres de Hłasko, l'illustration de couverture représente un poisson (Converti à Jaffa, une allusion à la fois au fait que l'action se situe dans une ville portuaire et à la symbolique chrétienne) et une valise (La mort du deuxième chien, l'histoire de deux vagabonds-escrocs). Pour finir, nous noterons que malgré sa ligne éditoriale, aucune des couvertures de Mirobole ne comporte d'éléments explicitement exotiques rattachables à l'origine périphérique des auteurs.

\section{AGULLO}

La politique éditoriale d'Agullo est aussi très clairement exprimée, mais plutôt que d'accentuer la périphéricité - un choix qui serait problématique car la maison publie également des auteurs français et américains -, l'éditeur explique son choix de titres uniquement par leur fonction anti-xénophobe. Ainsi, la maison veut « abolir les frontières » et se voit comme « le porte-voix d'auteurs d'ici et d'ailleurs », qui choisit des livres s'inscrivant « dans un monde où la curiosité et l'appétence de l'autre sont les meilleurs remèdes contre la peur et l'ignorance $»^{27}$. L'offre est divisée en deux collections : «Agullo Fiction » et « Agullo Noir ». Les cinq titres polonais font tous partie de la collection dédiée au polar et comprennent trois romans de Wojciech Chmielarz (Pyromane, La Ferme aux poupées et La Colombienne) et deux de Magdalena Parys (188 mètres sous Berlin et Le Magicien). Les notes de la quatrième de couverture consistent presque entièrement en des résumés sans références extradiégétiques. Les notices biographiques sont aussi assez neutres et présentent les auteurs principalement à travers le prisme des prix littéraires - nationaux dans le cas de Chmielarz (« il a été nominé trois fois au prestigieux prix du Gros Calibre, récompensant les meilleurs polars polonais ») et internationaux dans celui de Parys ( en 2019 elle a publié Le Magicien, qui a reçu le Prix de Littérature de l'Union européenne »). Les seules touches d'exotisme apportées par l'éditeur résident dans les citations des œuvres reproduites dans leur version originale (ici en polonais, mais le concept est utilisé pour plusieurs autres livres) sur le site web, à côté des résumés.

Le graphisme d'Agullo est, comme nous l'avons signalé, l'œuvre de Sean Habig, déjà connu pour sa collaboration avec Mirobole. Il propose, pour les couvertures, un concept toujours minimaliste et symbolique : il s'agit systématiquement d'un fond monochrome représentant un unique objet en blanc, le plus souvent sous la forme d'un contour sans détails et parfois un peu flou, qui se rapproche d'un cliché radiographique. Pour certains titres, ce procédé semble construire une

$27<\mathrm{http}: / /$ www.agullo-editions.com/\#collections $>$ [consulté le 30.04.2020]. 
métaphore plus ou moins sophistiquée, mais nous remarquons, pour les œuvres des auteurs polonais, un symbolisme explicitement lié à l'intrigue et parfois même au titre. Les couvertures des deux romans de Parys montrent trois cadres de tableaux (188 mètres sous Berlin, qui raconte l'histoire de la construction d'un tunnel entre Berlin-Ouest et la partie est de la ville aux fins d'un trafic d'œuvres d'art) et un appareil photographique cassé (Le Magicien, dont l'une des trames tourne autour de la mort d'un journaliste et reporter photographe). Les couvertures des trois romans de Chmielarz sont encore plus explicites : elles montrent un cocktail Molotov (Pyromane), une poupée morcelée (La Ferme aux poupées, dans une référence aussi bien au titre qu'à la scène du crime décrite dans le livre) et un rasoir entouré de poudre blanche (Colombienne, dont l'intrigue inclut le motif du narcotrafic en Colombie). Le lecteur potentiel reçoit ainsi, surtout avec Chmielarz, un iconotexte fort, sans trop d'ambiguïté, qui rappelle le graphisme simplifié des couvertures illustrées du roman populaire ${ }^{28}$. Néanmoins, tout comme dans le cas de Mirobole, les couvertures ne signalent aucunement la périphéricité des auteurs.

\section{CONCLUSION}

Comme l'a constaté Zofia Bobowicz - traductrice et rédactrice chez plusieurs éditeurs français, responsable de collections dédiées à l'Europe centrale et orientale — dans le cas des traductions de langues de l'Europe médiane « le principal problème consiste à trouver des lecteurs intéressés par cette littérature », car cette partie du continent « est un monde qui demeure toujours assez éloigné du lecteur français $»^{29}$. Les exemples des quatre maisons d'édition étudiées ci-dessus nous montrent que les éditeurs montrent rarement leurs auteurs et les œuvres de cette région par le prisme de leur périphéricité.

Considérant que l'éditeur qui publie des traductions « concourt à la création de l'image d'une culture autre dans la culture d'accueil » aussi bien « par la décision d'introduire l'œuvre traduite dans son catalogue » que par « la façon de la présenter au lecteur $»^{30}$, nous pouvons formuler l'hypothèse que l'image de la culture polonaise qui émerge du paratexte des livres analysés dans le présent article semble libre d'exotisation, procédé qui, dans le cas d'un transfert interculturel entre des périphéries et un centre, est toujours quelque peu marginalisant et condescendant.

${ }^{28}$ Cf. A. Audureau, « Étude des couvertures de la série des Fantômas dessinées par Gino Starace entre 1911 et $1913 »$, Belphégor 11-1, 2013 (<http://journals.openedition.org/belphegor/110> [consulté le 30.04.2020]).

29 Z. Bobowicz, «Traduction et marché du livre. Entretien réalisé par Joanna Nowicki », Hermès. La Revue 3(49), 2007, pp. 193-196.

30 E. Skibińska, «Les quatrièmes de couverture comme lieu d'inscription d'une représentation de la littérature traduite : romans canadiens d'expression française en traduction polonaise (2000-2016) », Między Oryginatem a Przektadem 3(45), 2019, p. 119. 
La question de l'efficacité d'un tel procédé en termes de marketing éditorial reste ouverte : pour y répondre exhaustivement, il faudrait que nous puissions accéder aux données concernant les ventes des titres analysés. Une autre direction de recherche qui pourrait ajouter des arguments pour ou contre l'efficacité des démarches entreprises par les éditeurs en ce sens serait d'étudier la réception des œuvres dont le paratexte est brièvement commenté ci-dessus pour voir si les lecteurs ont accepté ou non la vision qui y était proposée. Ceci, évidemment, nécessiterait une vaste étude approfondie dépassant le cadre d'un simple article.

\title{
DOES PERIPHERALITY SELL? \\ SOME COMMENTS ON THE EDITORIAL PERITEXTS OF POLISH NOVELS TRANSLATED INTO FRENCH
}

\begin{abstract}
The position of Polish literature in France is strictly marginal and is thus an excellent example of the relationship between the centre of the European book market and its periphery, regulated by dominant languages and characterised by an imbalance in the exchange of cultural goods.

Such a situation makes it possible for French publishers to use the peripherality category as a marketing tool in order to draw the attention of potential readers to books from a distant and unfamiliar region.

The article attempts to examine whether and how publishers highlight the peripheral nature of Polish literature in the paratexts of published translations. The research corpus consists of books published between 2008 and 2018 by four publishers: Noir sur Blanc, Actes Sud, Mirobole, and Agullo, who are among the most active entities importing Polish literature to France in the studied period.
\end{abstract}

Key words: book market, peripherality, exoticisation, paratext, book marketing. 